\title{
Electrochemical Features of Symmetric and Asymmetric Supercapacitors Based on Nanostructured Mn-CuO Electrodes
}

\author{
R. SURESH ${ }^{1 *}$, K. TAMILARASAN ${ }^{2}$ and D. SENTHIL VADIVU ${ }^{3}$ \\ ${ }^{1}$ Department of Physics, Sri Eshwar College of Engineering, Kondampatti, Kinathukadavu, \\ Coimbatore-641202, Tamil Nadu, India. \\ 2Department of Physics, Kongu Engineering College, Erode-638 052, Tamilnadu, India. \\ ${ }^{3}$ Department of Chemistry, Sree Saraswathi Thyagaraja College-642 003, Pollachi, India. \\ ${ }^{*}$ Corresponding author E-mail: suresh.r@sece.ac.in \\ http://dx.doi.org/10.13005/ojc/340648
}

(Received: September 11, 2018; Accepted: November 03, 2018)

\begin{abstract}
Progress in material science has unearthed a number of options that offer great advantages for nanostructured electrode materials which enable supercapacitors to operate efficiently. Present work involves fabrication of symmetric and asymmetric type supercapacitor devices utilizing $\mathrm{Mn}-\mathrm{CuO}$ nanostructures and activated carbon (AC) as electrode materials and subsequent investigation on their supercapacitive performance in $2 \mathrm{M} \mathrm{KOH}$ aqueous electrolyte. The asymmetric supercapacitor device (Mn-CuO // 2M KOH // AC) demonstrate a specific capacitance of $72 \mathrm{Fg}^{-1}$ at a current density of $0.5 \mathrm{Ag}^{-1}$. The cyclic stability test of this device performed at a current density of $10 \mathrm{Ag}^{-1}$ reveals a capacitance retention of $71 \%$ of its initial value over 300 charge-discharge cycles. In addition, this device exhibits an energy density of $7.4 \mathrm{Whkg}^{-1}$ and a power density of $127 \mathrm{Wkg}^{-1}$.
\end{abstract}

Keywords: Nanostructured electrodes, supercapacitors, aqueous electrolyte, energy density, cyclic stability, specific capacitance.

INTRODUCTION

Access to clean and affordable energy is an essential element for societal and economic development. In recent years, supercapacitors (SC) with their enhanced power capabilities, have attracted escalating attention among the research community due to their potential as promising energy storage devices for the future energy technology.

Since, SCs combine the energy storage properties of batteries with the power discharge characteristics of capacitors, they possess attractive features such as high power density, faster chargedischarge cycling, long cycle life, zero maintenance, environment friendly nature, enhanced safety and better endurance. Complementing with battery, supercapacitors are the appropriate choice for high demand vehicles like Hybrid Electric Vehicles (HEV) and find diversified industrial applications including portable electronic gadgets, cell phone base stations, digital cameras, aircraft, power tools and smart grids. ${ }^{1,2}$

This is an Open Access article licensed under a Creative Commons license: Attribution 4.0 International (CC- BY). Published by Oriental Scientific Publishing Company @ 2018 
Usually SCs consist of activated carbon as electrodes and are separated by polymeric separators with an organic electrolyte. ${ }^{3}$ Organic electrolytes provide higher potential window up to $4 \mathrm{~V}$, however it possess few disadvantages like low ionic conductivity, high cost and complicated purification process. Aqueous electrolytes with advantageous features like, higher ionic conductivity and low cost, ${ }^{4,5,6}$ offer SCs appropriate features for commercial exploitation. At present, this technology is rolling towards better understanding of charge storage mechanism and the discovery of superior electrode materials to realize SCs with enhanced performance.

Based on the type of electrodes involved for charge storage process, SCs are generally classified as symmetric type supercapacitors and asymmetric type supercapacitors. In symmetric type, both the positive and negative electrodes contain same type of electrode active material. The asymmetric type supercapacitor integrates a battery type electrode with a capacitor type electrode and hence could provide both the advantages of a battery (higher energy) and a capacitor (higher rate and power). ${ }^{7}$

\section{EXPERIMENTAL}

\section{Material synthesis - precipitation method}

Precipitation method involves creation of a solid in a solution by the chemical reaction that occurs between the soluble reactants in a liquid solution. Typical synthesis of $\mathrm{CuO}$ nanostructures involve dissolving $1.812 \mathrm{~g}$ of copper nitrate $\left(\mathrm{Cu}\left(\mathrm{NO}_{3}\right)_{2} \cdot 3 \mathrm{H}_{2} \mathrm{O}\right)$ in $250 \mathrm{~mL}$ of double distilled water under constant stirring. To the stirred solution, $2 \mathrm{~mL}$ of ethylene glycol and $0.09 \mathrm{~g}$ of disodium citrate were added followed by addition of $2 \mathrm{M} \mathrm{NaOH}$ drop by drop to make the $\mathrm{pH}$ of the solution as 10 . Homogenous mixture of the solution is obtained by stirring the solution for $5 \mathrm{~h}$ and aged for 2 days. The precipitate that formed remains in the suspension and were separated by centrifugation. The samples were collected and dried overnight at $80^{\circ} \mathrm{C}$ to get the final product. Exploiting the same procedure, $\mathrm{Mn}-\mathrm{CuO}$ nanostructures were obtained by adding manganese nitrate to the solution during the initial stage and adopting the subsequent steps as indicated earlier.

\section{Fabrication of symmetric and asymmetric} supercapacitors

$\mathrm{Mn}-\mathrm{CuO}$ nanostructures (5 wt\% of $\mathrm{Mn}$ doped $\mathrm{CuO}$ ) were utilized as positive and negative electrodes to construct symmetric type supercapacitor as they possess better supercapacitive properties (specific capacitance of $625 \mathrm{Fg}$. 1. better cyclic stability of over 2000 cycles besides exhibiting charge transfer resistance of $1 \Omega$ ) as revealed by suresh et al.,.8

The asymmetric type supercapacitor was fabricated utilizing $\mathrm{Mn}-\mathrm{CuO}$ nanostructure as one electrode and activated carbon (Sigma-Aldrich) as the other electrode with non-woven cloth as separator in both forms.

\section{Electrochemical characterization}

Utilizing $\mathrm{CHI} 660 \mathrm{D}$ electrochemical workstation ( $\mathrm{CH}$ Instruments), electrochemical measurements were performed at room temperature to determine the specific capacitance, power density and energy density using the mass of the active material present in the electrode $(1 \mathrm{mg})$.

\section{RESULTS AND DISCUSSION}

\section{Symmetric supercapacitor (Mn-CuO // 2M KOH// $\mathrm{Mn}-\mathrm{CuO})$}

The fabrication schematic of the symmetric type device employing $\mathrm{Mn}-\mathrm{CuO}$ nanostructures as electrode materials is shown in Figure 1.

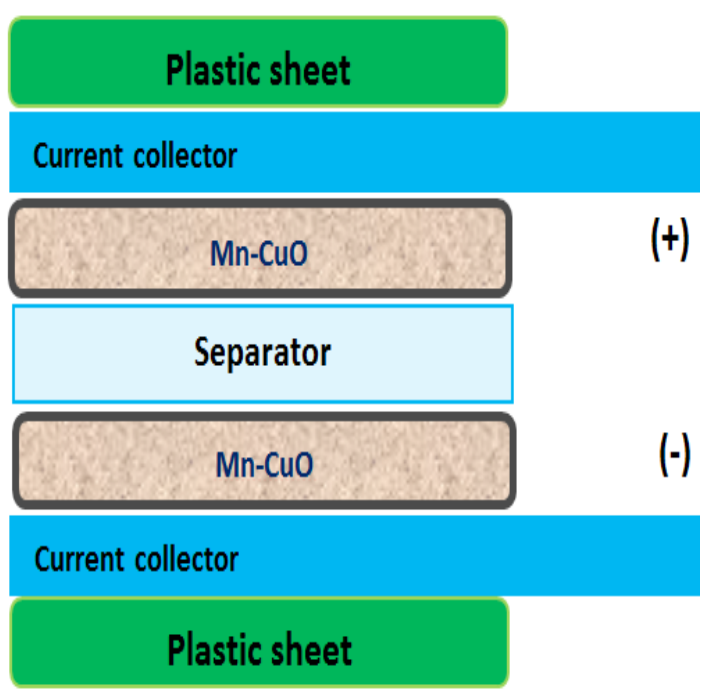

Fig. 1. Fabrication schematic of $\mathrm{Mn}-\mathrm{CuO} / / 2 \mathrm{M} \mathrm{KOH} / /$ $\mathrm{Mn}-\mathrm{CuO}$ symmetric supercapacitor device 
To investigate the electrochemical performance of $\mathrm{Mn}-\mathrm{CuO}$ nanostructure electrodes in a full cell capacitor, the cyclic voltammetry measurements were performed with $\mathrm{Mn}-\mathrm{CuO} / / 2 \mathrm{M}$ $\mathrm{KOH} / / \mathrm{Mn}-\mathrm{CuO}$ symmetric supercapacitor within the potential range from $0 \mathrm{~V}$ to $0.6 \mathrm{~V}$ using $2 \mathrm{M} \mathrm{KOH}$ aqueous solution as the electrolyte. Due to the better electrical conductivity and ionic mobility of $\mathrm{KOH}$ electrolyte, it is preferred for the present study.

Figure 2 (a) represent the CV curves measured at various scan rates. The quasirectangular shape of the $\mathrm{CV}$ curves without distinct redox peaks confirms the good capacitive nature of this symmetric device.
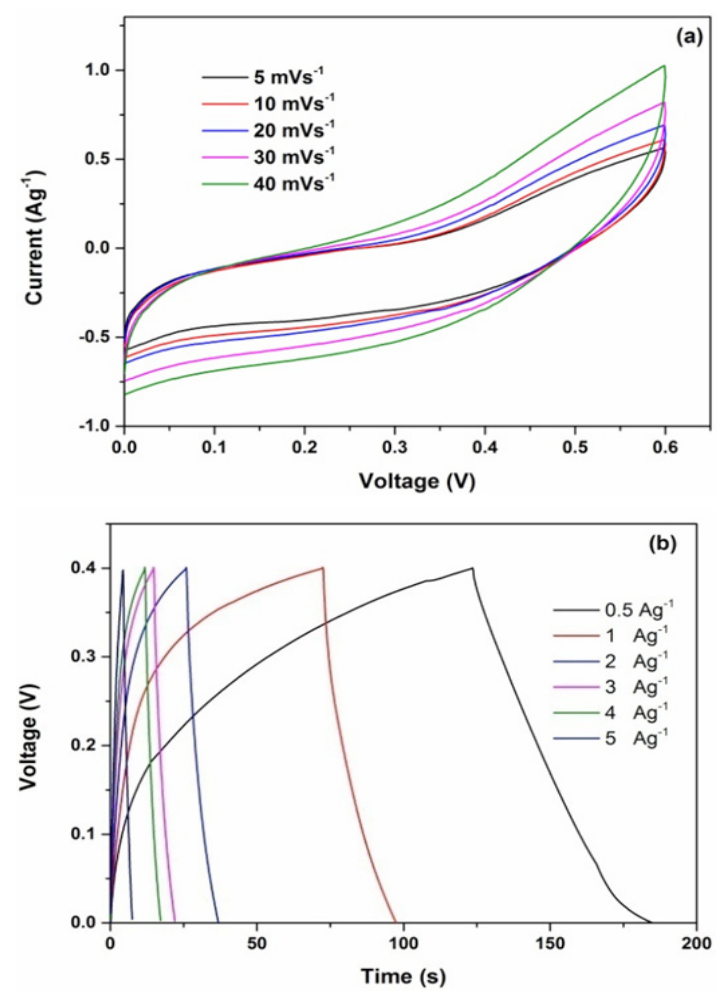

Fig. 2. (a) CV curves of symmetric supercapacitor (Mn-CuO // 2M KOH// Mn-CuO) at various scan rates (b) GCD curves of symmetric supercapacitor (Mn-CuO//2M $\mathrm{KOH} / / \mathrm{Mn}-\mathrm{CuO}$ ) at various current densities

In addition, Galvanostatic ChargeDischarge (GCD) measurements were carried out to derive the specific capacitance and to examine the sustainability of this material as supercapacitor electrodes at higher current rates. The GCD profiles with different current densities as presented in Fig. 2 (b) indicate the symmetrical charge and discharge which confirms the better reversibility and capacitive performance of the symmetric device. The GCD curves reveals specific capacitance values of $57,44,37,32$,
$28,25 \mathrm{Fg} .1$ at current densities of $0.5,1,2,3,4,5 \mathrm{Ag}^{-1}$ respectively. The variation of specific capacitance with current density is presented in Figure 3.

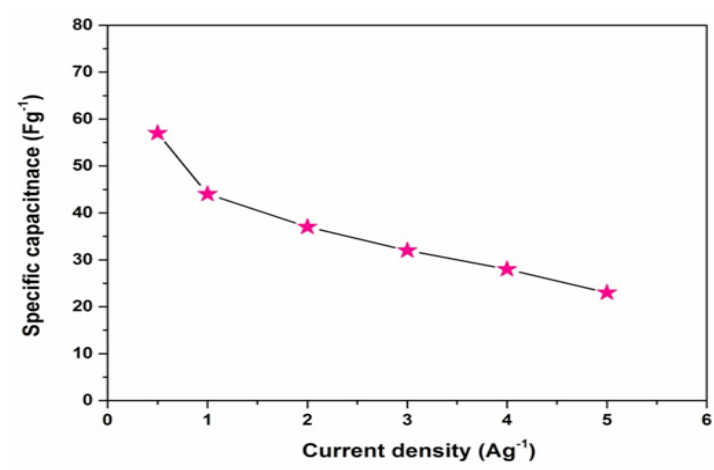

Fig. 3. Variation of specific capacitance with current densities for symmetric supercapacitor (Mn-CuO// 2M KOH// Mn-CuO)

For commercial exploitation, the cyclic stability is a critical parameter that confirms the stability and life of the supercapacitor. Chargedischarge cyclic stability test were performed for $\mathrm{Mn}-\mathrm{CuO} / / 2 \mathrm{M} \mathrm{KOH} / / \mathrm{Mn}-\mathrm{CuO}$ symmetric supercapacitor up to 300 cycles at a current density of $10 \mathrm{Ag}^{-1}$ to analyse its stability. It is obvious from Fig. 4 (a), the symmetric device retains $51 \%$ of its initial capacitance over 300 cycles. Further, the device shows an energy density and a power density of $5.8 \mathrm{Whkg}^{-1}$ and $128 \mathrm{WKg}^{-1}$ respectively.
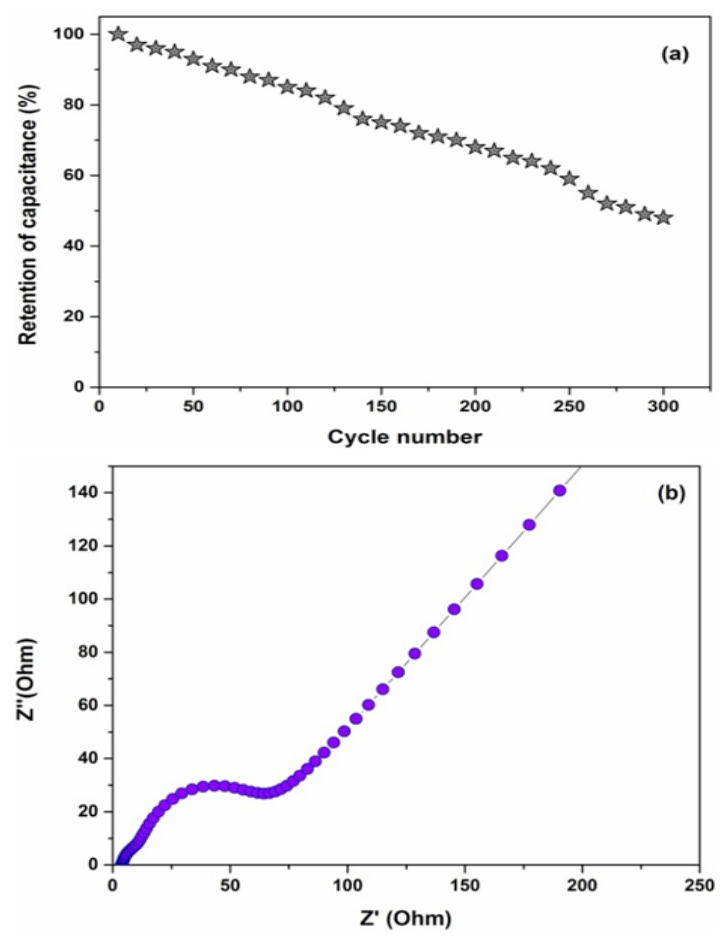

Fig. 4 (a) Cyclic stability test (b) Nyquist plot for symmetric supercapacitor in $\mathbf{2} \mathrm{M} \mathrm{KOH}$ aqueous solution 
The Electrochemical Impedance Spectroscopy (EIS) measurements were performed to get better insight of the resistive and capacitive elements involved with this symmetric device. Fig. 4 (b) demonstrates the Nyquist plot of $\mathrm{Mn}-\mathrm{CuO}$ //2M KOH//Mn-CuO symmetric supercapacitor which consists of a semicircle at higher frequency area and long tail in the lower frequency area. The EIS data has been fit to a modified Randles circuit shown in Fig. 5. The X-intercept on real axis of the plot is the combined resistance of electrolyte and the internal resistance of electrode $\left(R_{s}\right)$.

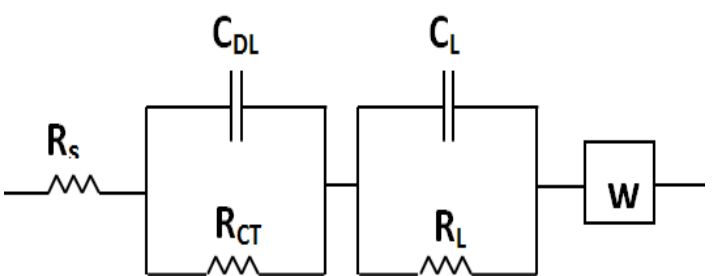

Fig. 5. Equivalent circuit model used for fitting ESI data

The diameter of the semicircle in the plot depicts the charge transfer resistance $\left(R_{c t}\right)$ due to Faradaic reactions and double layer capacitance $\left(C_{D L}\right)$ at electrode-electrolyte interface. Further, a charge transfer resistance of $63 \Omega$ was observed from the plot. An ideal polarizable capacitance exhibits a line nearly parallel to the imaginary axis with mass capacitance $\left(\mathrm{C}_{L}\right)$ at low frequencies. ${ }^{9}$ This resistance is named as leakage resistance $\left(R_{L}\right)$ and connected parallel to $C_{L}$. The change from high frequency semicircle to low-frequency tail is indicated by Warburg element (W).

\section{Asymmetric Supercapacitor (Mn-CuO//2M KOH//AC)}

A supercapacitor consists of metal oxide based positive electrode and carbon based negative electrode is highly attractive towards achieving higher energy and power densities. To realize the same, a battery type electrode (Mn-CuO) and a capacitor type electrode made of activated carbon (AC) are stacked in a single package to obtain an asymmetric supercapacitor. The fabrication schematic of $\mathrm{Mn}-\mathrm{CuO} / / 2 \mathrm{M} \mathrm{KOH//AC}$ asymmetric device is presented in Fig. 6. The asymmetric type device comprises of $\mathrm{Mn}-\mathrm{CuO}$ and activated carbon as electrodes and a non-woven cloth as a separator. To understand the electrochemical behaviour of $\mathrm{Mn}-\mathrm{CuO}$ nanostructure based asymmetric device, Cyclic Voltammetry (CV) investigations were made.

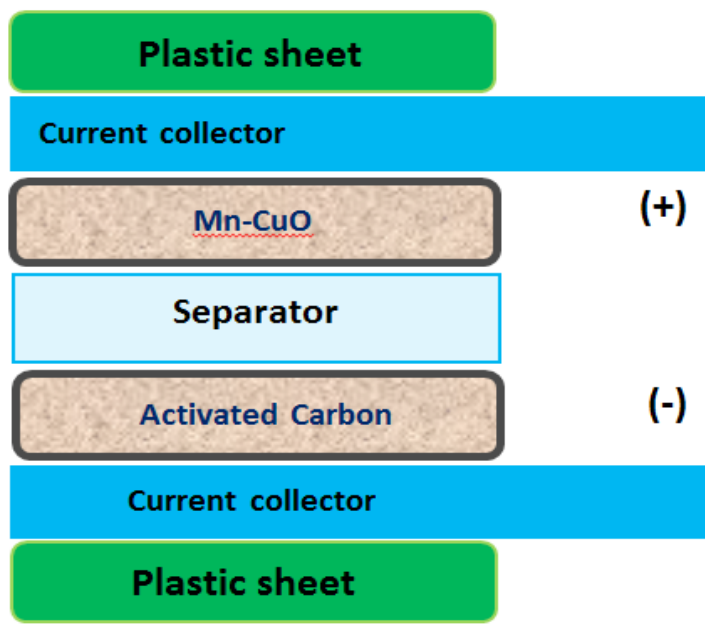

Fig. 6. Schematic illustration of fabrication of asymmetric supercapacitor (Mn-CuO// 2M KOH //AC)
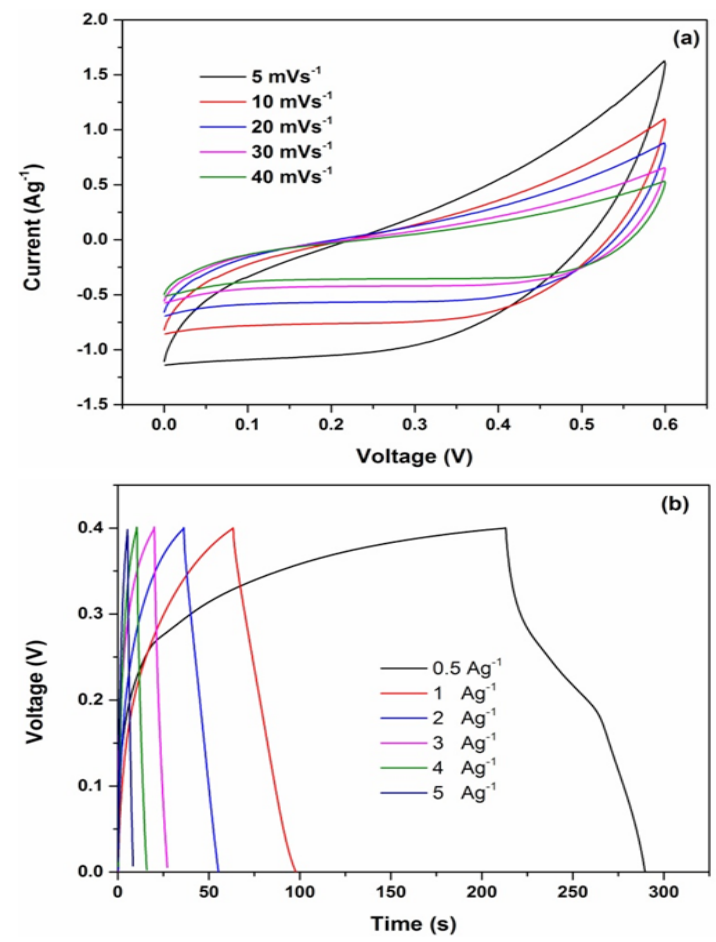

Fig. 7. (a) CV curves of Mn-CuO // 2M KOH// AC asymmetric supercapacitor device at different scan rates (b) GCD curves of $\mathrm{Mn}-\mathrm{CuO} / / 2 \mathrm{M} \mathrm{KOH} / / \mathrm{AC}$ asymmetric supercapacitor device at different current densities

The CV curves of the $\mathrm{Mn}-\mathrm{CuO} / / 2 \mathrm{M} \mathrm{KOH} / / \mathrm{AC}$ asymmetric supercapacitor device at different scan rates recorded in $2 \mathrm{M} \mathrm{KOH}$ aqueous electrolyte are shown in Fig. 7 (a). The near rectangular shape of the CV curve for a potential window between $0 \mathrm{~V}$ and $0.6 \mathrm{~V}$ indicate a good charge- discharge capacity of the device. The Galvanostatic Charge- 
Discharge (GCD) investigations for this asymmetric device were carried out to further understand its supercapacitive features. The triangular shape of the charge-discharge curves as presented in Fig. 7 (b) shows better symmetry and the linear slopes with various current densities demonstrate the good capacitive features of the device. The variation of specific capacitance with different current densities is presented in Figure 8.

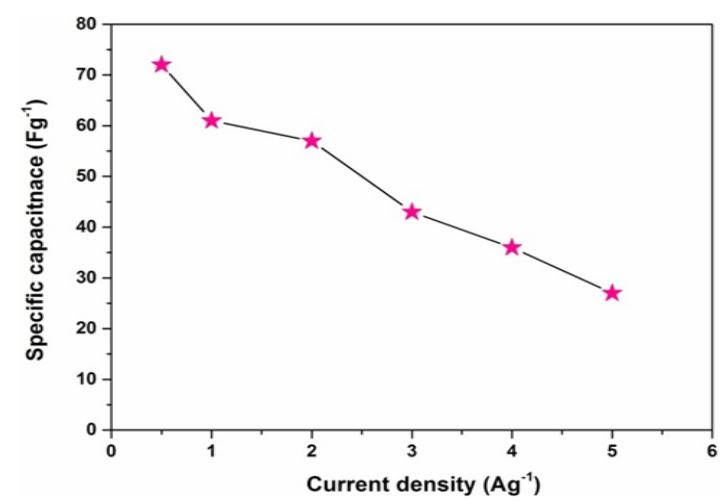

Fig. 8. Variation of specific capacitance with current densities for $\mathrm{Mn}-\mathrm{CuO} / / 2 \mathrm{M} \mathrm{KOH//} \mathrm{AC}$ asymmetric supercapacitor

From the discharge curves, the specific capacitance of the $\mathrm{Mn}-\mathrm{CuO} / / 2 \mathrm{M} \mathrm{KOH} / / \mathrm{AC}$ asymmetric supercapacitor device at current densities of $0.5,1,2$, $3,4,5 \mathrm{Ag}^{-1}$ are $72,61,57,43,36,27 \mathrm{Fg}^{-1}$ respectively. Fig. 9 (a) demonstrates the cyclic stability test of Mn$\mathrm{CuO} / / 2 \mathrm{M} \mathrm{KOH} / / \mathrm{AC}$ asymmetric device at a current density of $10 \mathrm{Ag}^{-1}$. This device retains $71 \%$ of its initial capacitance over 300 charge-discharge cycles which confirms better stability as compared to the $\mathrm{Mn}-\mathrm{CuO}$ nanostructure based symmetric device. The enhanced stability can be attributed to the presence of activated carbon electrode in the asymmetric type device. In addition, this device exhibits an energy density of 7.4 $\mathrm{Whkg}^{-1}$ and a power density of $127 \mathrm{WKg}^{-1}$.

The Nyquist plot of $\mathrm{Mn}-\mathrm{CuO} / / 2 \mathrm{M} \mathrm{KOH//AC}$ asymmetric supercapacitor device as displayed in Fig. 9 (b) provides a charge transfer resistance $\left(R_{c t}\right)$ of $48 \Omega$ for this asymmetric device. The lower value of charge transfer resistance $\left(R_{c t}\right)$ in asymmetric device is due to the presence of highly conductive activated carbon electrode which considerably improves the electrochemical features like specific capacitance, stability and energy density of the device.
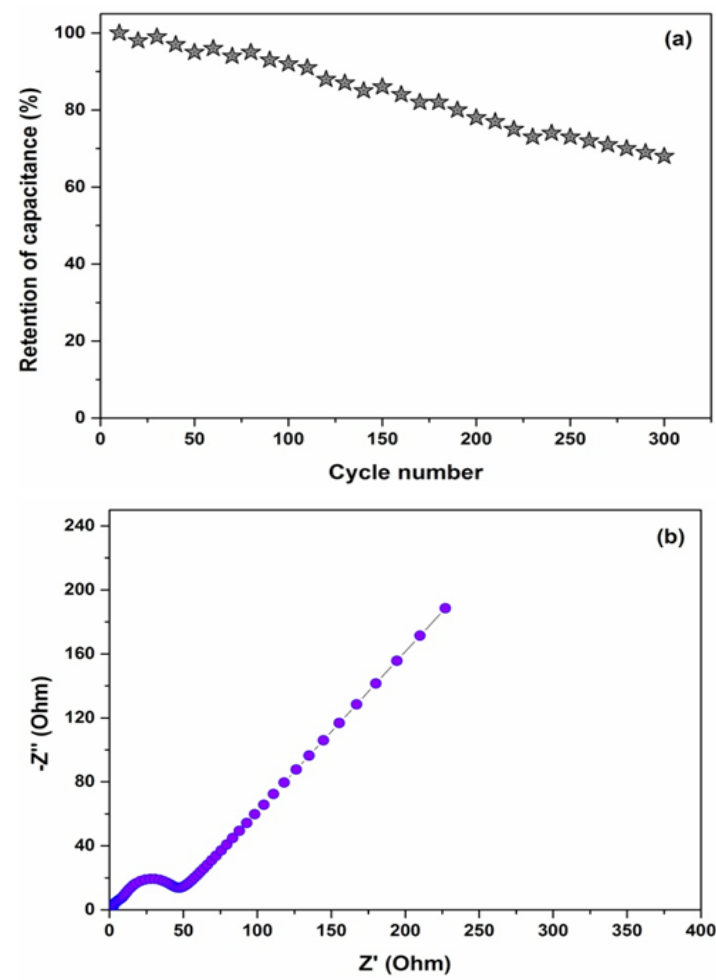

Fig. 9. (a) Cyclic stability test for asymmetric supercapacitor at $10 \mathrm{Ag}^{-1}$ (b) Nyquist plot of asymmetric supercapacitor in $2 \mathrm{M} \mathrm{KOH}$ aqueous solution

\section{CONCLUSION}

Asymmetric and symmetric type supercapacitor devices based on $\mathrm{Mn}-\mathrm{CuO}$ nanostructures and activated carbon as electrodes in $2 \mathrm{M} \mathrm{KOH}$ aqueous electrolyte have been assembled and investigated for their electrochemical performance. The $\mathrm{Mn}-\mathrm{CuO} / / 2 \mathrm{M} \mathrm{KOH} / / \mathrm{AC}$ asymmetric supercapacitor retains $71 \%$ of initial capacitance after 300 cycles. Moreover, the asymmetric type device exhibits a specific capacitance of $72 \mathrm{Fg}^{-1}$ along with an energy density of 7.4 $\mathrm{Whkg}^{-1}$ indicating its edge over symmetric type device $\left(57 \mathrm{Fg}^{-1}, 5.8 \mathrm{Whkg}^{-1}\right)$ in supercapacitor applications.

\section{ACKNOWLEDGEMENT}

The authors would like to thank Sri Eshwar College of Engineering, Coimbatore, Tamilnadu, India for the administrative support and Kongu Engineering College, Perundurai, Coimbatore, Tamilnadu, India for the research facilities. 


\section{REFERENCES}

1. Wu, L.; Hao, L.; Pang, B.; Wang, G.; Zhang, Y.; Li, X. Journal of Materials Chemistry A., 2017, 5, 4629-4637.

2. Hall, P.J.; Mirzaeian, M.; Fletcher, S.I.; Sillars, F.B.; Rennie, A.Y.R.; Shitta-Bey, G.O.; Wilson, G.; Cruden, A.; Carter, R. Energy and Environmental Science., 2010, 3, 1238-1251.

3. Burke, A. Electrochimica Acta., 2007, 53, 1083-1091.

4. Ghosh, A.; Lee, Y.H. Chem Sus Chem., 2012, 5, 480-499.

5. Beguin, F; Presser, V.; Balducci, A. Frackowiak, E. Advanced Materials., 2014,
26, 2219-2251.

6. Qu, Q.T.; Zhang, P.; Wang, B.; Chen, Y.H.;Tian, S.; Wu, Y.P.; Holze, R.J. Journal of Physical Chemistry C., 2009, 113, 14020-14027.

7. Chen, P.C.; Shen, G.Z.; Shi, Y.; Chen, H.T.; Zhou, C.W. ACS Nano., 2010, 4, 4403-4411.

8. Suresh, R.; Tamilarasan, K.; Senthil Vadivu, D. Digest Journal of Nanomaterials and Biostructures., 2016, 11, $795-803$.

9. Masarapu, C.; Zeng, H.F.; Hung, K.H.; Wei, B. ACS Nano., 2009, 3, 2199-2206.

10. Yan, J.; Liu, J.; Fan, Z.;Wei, T.; Zhang, L. Carbon., 2012, 50, 2179-2188. 